\title{
Classical $R$-matrix structure for the Calogero model
}

\author{
J.Avan M.Talon *
}

October 1992

\begin{abstract}
A classical $R$-matrix structure is described for the Lax representation of the integrable $n$-particle chains of Calogero-Olshanetski-Perelomov. This $R$ matrix is dynamical, non antisymmetric and non-invertible. It immediately triggers the integrability of the Type I, II and III potentials, and the algebraic structures associated with the Type $\mathrm{V}$ potential.
\end{abstract} Jussieu, F-75252 PARIS CEDEX 05 


\section{Introduction}

A general description of classically integrable systems of $n$ particles with two-body interactions $v\left(x_{i}-x_{j}\right)$ was undertaken in [1]. The authors described a set of five two-body potentials, respectively denoted I $\left(v(q)=q^{-2}\right)$, II $\left(v(q)=a^{2} \sinh ^{-2} a q\right)$, III $\left(v(q)=a^{2} \sin ^{-2} a q\right)$, IV $\left(v(q)=a \mathcal{P}\left(a q, \omega_{1}, \omega_{2}\right) ; \mathcal{P}\right.$ being the double-periodic Weierstrass function), $\mathrm{V}\left(v(q)=q^{-2}+g q^{2}\right)$. To this list can be added the Toda potential $v(q)=e^{-q}$, denoted VI. It must be noticed that I, II, III are particular limits of IV. These potentials were originally derived in [2].

To each simple Lie algebra and choice of one of these six potentials one can associate a classically integrable system (see Section 2). The simplest case is the $A_{n}$ algebra, for which the first non-trivial Hamiltonian of the hierarchy describes the original Calogero model:

$$
\mathcal{H}=\sum_{i=1}^{n} p_{i}^{2}+\sum_{i \neq j} v\left(q_{i}-q_{j}\right)
$$

$\left(p_{i}, q_{i}\right)$ being canonically conjugate variables for the $i$-th particle. The choice of Potential V leads to a model which was recently shown to be related to the collective theory of 2-dimensional strings [3].

This Hamiltonian system, and the others obtained from different choices of algebra (detailed in [1]), were shown to be integrable. Their dynamics is described by a Lax representation [4], and one is able to construct a set of Poisson-commuting Hamiltonians from this representation [6, 5]. However the associated algebraic structure of the Lax representation is not known; in particular the $R$-matrix describing the Poisson brackets of the Lax operator [7], known to exist from general arguments [8], has not yet been constructed.

Our main result is an explicit construction of the $R$-matrix for the $A_{n}$ models with potentials of the type I, II, III and V. The Lax operator reads:

$$
L \equiv \sum_{i=1}^{n} p_{i} e_{i i}+i \sum_{i \neq j} w\left(q_{i}-q_{j}\right) e_{i j} ; v\left(q_{i}-q_{j}\right) \equiv w^{2}\left(q_{i}-q_{j}\right)
$$

and its $R$-matrix reads (with $\left(e_{i j}\right)_{k l}=\delta_{i k} \delta_{j l}$ ):

$$
\begin{aligned}
R \equiv & \sum R^{i j k l} e_{i j} \otimes e_{k l}= \\
& -\sum_{i \neq j} \frac{w^{\prime}\left(q_{i}-q_{j}\right)}{w\left(q_{i}-q_{j}\right)} e_{i j} \otimes e_{j i}+\sum_{i \neq j} w\left(q_{i}-q_{j}\right) e_{i i} \otimes\left(e_{i j}-e_{j i}\right)
\end{aligned}
$$

Note that $\mathcal{H}$ in (11) is $\operatorname{Tr} L^{2}$, an ad-invariant function of the Lax matrix, hence is conserved.

The plan of this paper is as follows. In Section 2 we recall the main features of the Lax representation for the Calogero-Olshanetski-Perelomov models, and the 
current proofs of integrability. In Section 3 we derive directly the $R$-matrix (3) for the Lax matrix (2) and check that this leads to the correct $M$ matrix [1] in the Lax pair for the Hamiltonian $\operatorname{Tr} L^{2}$. In Section 4 we elaborate on the case of Potential V. Two "Lax matrices" $L^{+}$and $L^{-}$are introduced and the $R$-matrix structure allows an immediate interpretation of the angle-type variables $\operatorname{Tr}\left(L^{ \pm}\right)^{n}$ and action-type variables $\operatorname{Tr}\left(L^{+} L^{-}\right)^{n}$, and of the interesting algebraic structures arising in the explicit solution of this model, described in [9, 10]. Finally we give some concluding remarks on the extension to the generic potential IV, and other algebras, of this $R$-matrix formalism.

\section{The Lax representation and integrability of the C. O. P. model (Type I-IV)}

We shall briefly reproduce here results for the algebra $A_{n}$ described in detail in the review article [1]. Introducing an Ansatz for the Lax pair $(L, M)$ of the form:

$$
\begin{aligned}
L_{j k} & =p_{j} \delta_{j k}+i\left(1-\delta_{j k}\right) w\left(q_{j}-q_{k}\right) \\
M_{j k} & =\delta_{j k} \sum_{i \neq j} z\left(q_{i}-q_{j}\right)-\left(1-\delta_{j k}\right) y\left(q_{i}-q_{k}\right) \\
k, j & =1 \ldots n
\end{aligned}
$$

where $x$ is an odd function and $y, z$ are even functions, it is shown that the Lax equation $d L / d t=[L, M]$ reproduces the Hamiltonian equations for the two-body potential $v\left(q_{i}-q_{j}\right)$, provided one identifies:

$$
\begin{aligned}
v(q) & =w^{2}(q) \\
z(q)=\frac{w^{\prime \prime}(q)}{2 w(q)} & ; \quad y(q)=-w^{\prime}(q)
\end{aligned}
$$

and $w$ obeys the functional equation:

$$
w\left(q_{1}\right) w^{\prime}\left(q_{2}\right)-w\left(q_{2}\right) w^{\prime}\left(q_{1}\right)=w\left(q_{1}+q_{2}\right)\left(z\left(q_{2}\right)-z\left(q_{1}\right)\right)
$$

This functional equation admits four solutions [2, 11], one generic $\left(w(q)=a \mathrm{sn}^{-1}(a q)\right)$ corresponding to the Type IV potential, and three limits of this solution, obtained when one of the periods of sn is sent to $+i \infty\left(w(q)=a \sin ^{-1}(a q)\right.$, Type III; $w(q)=a \sinh ^{-1}(a q)$, Type II $)$ and finally $a$ sent to $0(w(q)=1 / q$, Type I $)$.

The Type $\mathrm{V}$ potential $v(q)=1 / q^{2}+q^{2}$ can be obtained from a modification of the Lax representation which will be discussed in Section 4. The Type VI potential $v(q)=e^{-q}$ leads to the open Toda chain. Although not belonging to the set defined by the Lax pair (四), it nevertheless has some related features which we shall comment upon in the conclusion. 
The Lax representation (14) is associated with the algebra $A_{n}$. A general formulation was discussed in [1], involving the introduction of general root systems $\Phi$. The two-body potential becomes:

$$
U(\vec{q})=\sum_{\alpha \in \Phi} g_{\alpha}^{2} v\left(q_{\alpha}\right) ; q_{\alpha}=(\alpha, \vec{q}) ; \vec{q} \equiv\left(q_{1}, \ldots q_{n}\right)
$$

The candidate Lax operator reads:

$$
L=\sum_{\alpha \in \Phi} g_{\alpha} w\left(q_{\alpha}\right) e_{\alpha}-\sum_{\alpha \in \Phi \text { simple }} p_{\alpha} h_{\alpha}
$$

We shall solely describe the details of the derivation for the algebra $A_{n}$ and say a few words in conclusion concerning a general algebraic setting.

Integrability a la Liouville does not automatically follows from the existence of the Lax representation. One needs to check that the conserved quantities $\operatorname{Tr}_{E} L^{n}$ have vanishing Poisson brackets [12]. When an $R$-matrix structure is known, this result is trivial [17]. The proof runs as follows:

$$
\begin{aligned}
& \left\{\operatorname{Tr}_{E}\left(L^{n}\right), \operatorname{Tr}_{E}\left(L^{m}\right)\right\}=\operatorname{Tr}_{E \otimes E}\left\{L^{n} \otimes L^{m}\right\} \\
& \quad=n m \operatorname{Tr}_{E \otimes E}\left\{L^{n} \otimes L^{m}\left([R, L \otimes \mathbf{1}]-\left[R^{\Pi}, \mathbf{1} \otimes L\right]\right)\right\} \\
& \quad=0 \text { by cyclicity of the trace. }
\end{aligned}
$$

However, without such an explicit structure, the previously proposed demonstrations relied on arguments of asymptotic behaviour [13, or computational devices using inverse scattering-type methods [11], or direct - and complicated- recursion procedures [5].

On the other hand, the Poisson commutation of the ad-invariant conserved quantities, whichever way it is proved, implies the existence of an $R$-matrix, a priori dynamical [8], which we are now going to construct.

\section{The $R$-matrix for type I-III}

The Poisson bracket structure for the Lax operator $L$ appearing in eq. (丑) is the following:

$$
\{L, L\}=\sum_{i \neq j} \frac{w^{\prime}\left(q_{i}-q_{j}\right)}{w\left(q_{i}-q_{j}\right)}\left(e_{i i} \otimes e_{i j}-e_{i j} \otimes e_{i i}\right)
$$

where $e_{i j}$ are the generators of $A_{n}$ in the fundamental representation.

A generic $R$-matrix structure reads [17, 7, 8]:

$$
\{L \otimes, L\}=[R, L \otimes \mathbf{1}]-\left[R^{\Pi}, \mathbf{1} \otimes L\right]
$$


$R$ belongs to the tensor algebra $A_{n} \otimes A_{n} ; R^{\Pi}$ is the operator obtained by exchanging the two terms in $A_{n} \otimes A_{n}$. Introducing the components:

$$
R \equiv \sum_{a, b, c, d} R^{a b c d} e_{a b} \otimes e_{c d}
$$

the Poisson bracket structure $(9-10)$ is equivalent to the system of overdetermined equations:

$$
\begin{aligned}
& \left(p_{i}-p_{a}\right) R^{a i c d}+\left(p_{c}-p_{d}\right) R^{c d a i}+\sum_{k}\left(\frac{R^{a k c d}}{q_{k}-q_{i}}-\frac{R^{k i c d}}{q_{a}-q_{k}}-\frac{R^{c k a i}}{q_{k}-q_{d}}+\frac{R^{k d a i}}{q_{c}-q_{k}}\right) \\
= & \delta_{a i}\left(\delta_{a c}-\delta_{a d}\right) \frac{w^{\prime}\left(q_{c}-q_{d}\right)}{w\left(q_{c}-q_{d}\right)}-\delta_{c d}\left(\delta_{a c}-\delta_{i c}\right) \frac{w^{\prime}\left(q_{a}-q_{i}\right)}{w\left(q_{a}-q_{i}\right)}
\end{aligned}
$$

In order to solve this system, we shall make a number of assumptions which will ultimately restrict us to considering only the potentials of type I to III.

$\underline{\text { Assumption } 1}$ : $R$ does not depend on $p_{i}$

This decouples (12) into two sets of equations. The first one is easily solved:

$$
\left(p_{i}-p_{a}\right) R^{a i c d}+\left(p_{c}-p_{d}\right) R^{c d a i}=0
$$

One gets:

$$
\begin{aligned}
R^{a i c d}=0 \forall a \neq i \neq c \neq d \quad & R^{c d a a}=R^{a c a d}=R^{a c d a}=0 \forall a \neq c \neq d \\
R^{a i i a}=-R^{\text {iaai }} \forall a \neq i \quad & R^{\text {aiii }}=R^{\text {iaii }}=0 \forall a \neq i
\end{aligned}
$$

This leaves as free parameters of $R: \quad R^{a a c d} ; R^{a a i i}, R^{a i a i}, R^{a i i a} ; R^{i i a i}, R^{i i i a} ; R^{i i i i}$. Plugging these residual terms into (12) leads to a new set of equations:

(a) $\left(R^{a a c d}-R^{i i c d}\right) w\left(q_{a}-q_{i}\right)=\left(R^{c c a i}-R^{d d a i}\right) w\left(q_{c}-q_{d}\right)$

(b) $R^{\text {ccii }}=R^{\text {ddii }} \forall c, d$

(c) $\left(R^{a d a d}+R^{a i a i}\right) w\left(q_{d}-q_{i}\right)+\left(R^{a a a d}-R^{i i a d}\right) w\left(q_{a}-q_{i}\right)$

$$
+\left(R^{a a a i}-R^{d d a i}\right) w\left(q_{d}-q_{a}\right)=0
$$

(d) $\left(R^{a d d a}-R^{a i i a}\right) w\left(q_{d}-q_{i}\right)+\left(R^{a a d a}-R^{i i d a}\right) w\left(q_{a}-q_{i}\right)$

$$
+\left(R^{a a a i}-R^{d d a i}\right) w\left(q_{d}-q_{a}\right)=0
$$

$(e)-\left(R^{d a d a}+R^{i a i a}\right) w\left(q_{d}-q_{i}\right)+\left(R^{a a d a}-R^{i i d a}\right) w\left(q_{a}-q_{i}\right)$

$$
+\left(R^{a a i a}-R^{d d i a}\right) w\left(q_{d}-q_{a}\right)=0
$$

(f) $\quad R^{a a c a}-R^{a a a c}+R^{c c a c}-R^{c c c a}=0$

(g) $-R^{i i i i}-R^{i a a i}+R^{a a i i}-R^{a i a i}=\frac{w^{\prime}\left(q_{a}-q_{i}\right)}{w\left(q_{a}-q_{i}\right)}$

(h) $\quad R^{i i i i}-R^{i a a i}-R^{a a i i}+R^{i a i a}=\frac{w^{\prime}\left(q_{a}-q_{i}\right)}{w\left(q_{a}-q_{i}\right)}$ 
This system actually admits a solution with a minimal number of non-zero parameters, obtained by attempting to trivialize a maximal number of the above equations. Namely one sets:

Assumption 2:

$$
R^{a a c d}=0 ; R^{c c i i}=0 ; R^{i i i i}=0 ; R^{a i a i}=0
$$

thereby trivializing $(a, b, f, g, h)$ and leaving two non-vanishing parameters:

$$
R^{i a a i}=\frac{w^{\prime}\left(q_{a}-q_{i}\right)}{w\left(q_{a}-q_{i}\right)} ; R^{a a d a}=-R^{a a a d}=c . w\left(q_{a}-q_{d}\right)
$$

provided (from $(d))$ that $g \equiv 1 / w$ obeys the functional equation:

$$
g(u) g^{\prime}(v)+g(v) g^{\prime}(u)=c \cdot g(u+v)
$$

This functional equation is obeyed by the $w$ (or $g$ ) functions corresponding to the potentials of Type I, II and III, but not Type IV, with the constant $c$ equal to 1; hence our two assumptions, although restrictive, finally lead us to the $R$-matrix structure for these three potentials :

$$
R=-\sum_{i \neq j} \frac{w^{\prime}\left(q_{i}-q_{j}\right)}{w\left(q_{i}-q_{j}\right)} e_{i j} \otimes e_{j i}+\sum_{i \neq j} w\left(q_{i}-q_{j}\right) e_{i i} \otimes\left(e_{i j}-e_{j i}\right)
$$

with $w(q)=1 / q($ Type I), $1 / \sin (q)($ Type II), $1 / \sinh (q)$ (Type III). This $R$-matrix is dynamical, non-antisymmetric and non-invertible.

From the form of the $R$-matrix (19) immediately follows the $M$ matrix of the Lax pair associated to any Hamiltonian of the hierarchy. In particular for $\mathcal{H}=$ $\operatorname{Tr}(L)^{n}$, one has $[7,8]$ :

$$
M=\operatorname{Tr}_{2}(R . d \mathcal{H})=\operatorname{Tr}_{2}\left(R .1 \otimes L^{n-1}\right)
$$

In the case $n=2$, when $\mathcal{H}$ is given by (1), one gets back the Olshanetski-Perelomov formula [1]:

$$
M=\sum_{i \neq j} \frac{w^{\prime}\left(q_{i}-q_{j}\right)}{w\left(q_{i}-q_{j}\right)} e_{i j}+\sum_{i \neq j} w^{2}\left(q_{i}-q_{j}\right) e_{i i}
$$

Moreover the remarkable identities which allowed to show the Poisson commutation of the eigenvalues of $L$ are naturally equivalent to the existence and structure of the $R$-matrix, which is after all a compact canonical way of formulating special Poisson bracket relations leading to integrability. 


\section{The Type V Potential}

A related Poisson structure can now be described for the Type $\mathrm{V}$ potential $v(q)=$ $q^{-2}+g q^{2}$. The Lax representation [15] is not isospectral; one introduces two Lax operators $L^{+}$and $L^{-}$, respectively:

$$
L^{ \pm}=L \pm i \omega \operatorname{Diag}\left(q_{i}\right) ; L \equiv L\left(w_{I} \equiv 1 / q\right) ; g \equiv \omega^{2}
$$

They obey modified Lax equations:

$$
\frac{d L^{ \pm}}{d t}=\left[L^{ \pm}, M\left(w_{I}\right)\right] \pm g L^{ \pm}
$$

from which one defines [9] angle-type variables $\operatorname{Tr}\left(L^{ \pm}\right)^{n}$, and action variables $\operatorname{Tr}\left(L^{+} L^{-}\right)^{n}$.

The Poisson algebra of these variables is a consequence of the $R$-matrix structure of $L^{+}$and $L^{-}$. One ends up with:

$$
\begin{aligned}
& \left\{L^{+} \otimes L^{+}\right\}=\left[R, L^{+} \otimes \mathbf{1}\right]-\left[R^{\Pi}, \mathbf{1} \otimes L^{+}\right] \\
& \left\{L^{-} \otimes L^{-}\right\}=\left[R, L^{-} \otimes \mathbf{1}\right]-\left[R^{\Pi}, \mathbf{1} \otimes L^{-}\right] \\
& \left\{L^{+} \otimes L^{-}\right\}=\left[R, L^{+} \otimes \mathbf{1}\right]-\left[R^{\Pi}, \mathbf{1} \otimes L^{-}\right] \\
& +i \omega\left(\sum_{i \neq j} e_{i j} \otimes e_{j i}+\sum_{k} e_{k k} \otimes e_{k k}\right)
\end{aligned}
$$

From (24, 25) it immediately follows that the angle variables $B_{n}^{ \pm} \equiv \operatorname{Tr}\left(L^{ \pm}\right)^{n}$ Poisson-commute when having the same \pm gradation. From (26) one also deduces, not so straightforwardly, that the conserved quantities $\operatorname{Tr}\left(L^{+} L^{-}\right)^{n}$ Poissoncommute. Precisely one has:

$\left\{L^{+} L^{-}, L^{+} L^{-}\right\}=$

$\left[R . \mathbf{1} \otimes L^{-}+\mathbf{1} \otimes L^{+} . R, L^{+} L^{-} \otimes \mathbf{1}\right]-\left[L^{+} \otimes \mathbf{1} . R^{\Pi}+R^{\Pi} . L^{-} \otimes \mathbf{1}, \mathbf{1} \otimes L^{+} L^{-}\right]$

$+i \omega 1 \otimes L^{+} . \Pi_{s l(n)} . L^{-} \otimes 1-i \omega L^{+} \otimes 1 . \Pi_{s l(n)} \cdot 1 \otimes L^{-}$

where $\prod_{s l(n)} \equiv \sum_{i \neq j} e_{i j} \otimes e_{j i}+\sum_{k} e_{k k} \otimes e_{k k}$. Incidentally the $R$-matrix structure in the first two terms of (27) is a nice example of a second Poisson structure canonically obtained from a first structure by a Sklyanin-type bracket [18].

Hence in computing the brackets $\left\{\operatorname{Tr}\left(L^{+} L^{-}\right)^{n}, \operatorname{Tr}\left(L^{+} L^{-}\right)^{m}\right\}$ the contribution from the commutators vanish, as usual in such computations, and one is left with:

$\left\{\operatorname{Tr}\left(L^{+} L^{-}\right)^{n}, \operatorname{Tr}\left(L^{+} L^{-}\right)^{m}\right\}=+i \omega L^{-}\left(L^{+} L^{-}\right)^{n-1} \otimes\left(L^{+} L^{-}\right)^{m-1} L^{+} . \Pi_{s l(n)}$

$-i \omega\left(L^{+} L^{-}\right)^{n-1} L^{+} \otimes L^{-}\left(L^{+} L^{-}\right)^{m-1} \cdot \Pi_{s l(n)}$

Clearly the r.h.s. of (28) is an odd function of $\omega$ since $L^{+} \leftrightarrow L^{-}$when $\omega \leftrightarrow$ $-\omega$. However $\operatorname{Tr}\left(L^{+} L^{-}\right)^{n}=\operatorname{Tr}\left(L^{-} L^{+}\right)^{n}$ by cyclicity and therefore the l.h.s. of 
(28) is an even function of $\omega$. It follows that both sides must be equal to 0 . Hence the conserved quantities $\operatorname{Tr}\left(L^{+} L^{-}\right)^{n}$ Poisson-commute and the potential V is Liouville-integrable.

Some comments are in order at this point. The Type-V potential has received a lot of attention recently. It is a discretized version of the collective field theory for two-dimensional strings [3]. Clearly the diagonalization of this field theory, undertaken in [10], and the underlying $w_{\infty}$ algebra of eigen-operators, is a natural consequence of the algebraic structure (24 - 26). The operators $B_{n}^{ \pm}$are in fact "shifted" action variables of the potential type I ; they realize through the crossed terms of (26) an algebra of oscillator type, which then generates naturally the $w_{\infty}$ algebra [19]. Moreover they are natural angle-type variables of the Type V potential, hence they diagonalize the Hamiltonian $\operatorname{Tr}\left(L^{+} L^{-}\right)$.

Finally a recent resolution of the Calogero model [16] relies on the existence of a very similar algebra of covariant derivatives a la Knizhnik-Zamolodchikov. However an exact relation between these two algebras is not yet available.

\section{Conclusion}

As we have mentioned, this construction does not realize an $R$-matrix for generic (Type IV) elliptic potentials, due to the functional constraint (18). From the derivation in Section 3, it is clear that the $R$-matrix must then acquire supplementary terms, for instance $R^{i i k l}$, making an eventual complete resolution rather complicated. We shall not attempt to discuss it any further here.

The extension to other algebras sounds more promising. As indicated, the generic Lax operator takes the form:

$$
L=\sum_{\alpha \in \Phi} g_{\alpha} w\left(q_{\alpha}\right) e_{\alpha}-\sum_{\alpha \in \Phi \text { simple }} p_{\alpha} h_{\alpha}
$$

From our previous results, and allowing for general Chevalley relations

$$
\left[e_{\alpha}, e_{\beta}\right]=N_{\alpha \beta} e_{\alpha+\beta}
$$

we expect for the Type I-III potential an $R$-matrix of the form:

$$
R=\sum_{\alpha \in \Phi} c(\alpha) \frac{w^{\prime}(\alpha(q))}{w(\alpha(q))} e_{\alpha} \otimes e_{-\alpha}+\sum_{\alpha \in \Phi} d(\alpha) w(\alpha(q)) h_{\alpha} \otimes e_{\alpha}
$$

Here $c(\alpha), d(\alpha)$ are constant $(q)$ functions of the particular root $\alpha$.

Finally it is interesting to note that Type VI potential $\left(x(q)=e^{q}\right)$, although having a very different Lax formulation, has an associated $R$-matrix [20] reminding of (19). Provided one eliminates the second term, and replaces $x(q)$ by $e^{q}$ in (19), one ends up with:

$$
R=\sum_{i>j} e_{i j} \otimes e_{j i}-\sum_{i<j} e_{i j} \otimes e_{j i} \equiv R^{\text {Toda }}
$$




\section{Acknowledgements}

We wish to thank Olivier Babelon, John Harnad, Alexei Reiman, and Claude Viallet for fruitful discussions.

\section{References}

[1] M.A. Olshanetsky, A.M. Perelomov ; Phys. Rep. 71 (1981), 313.

[2] F. Calogero ; Lett. Nuov. Cim. 13 (1971), 411; ibid. 16 (1976), 22, 77.

[3] J. Avan, A. Jevicki ; Phys. Lett B266 (1991), 35; ibid. B272 (1991), 17.

[4] P.D. Lax ; Comm. Pure Appl. Math. 81 (1968), 467.

[5] S. Wojciechowski ; Lett. Nuov. Cim. 18 (1977), 103.

[6] A.M. Perelomov ; Preprint ITEP 27 (1976).

[7] M.A. Semenov-Tjan-Shanskii ; Funct. Anal. Appl. 17 (1983),259.

[8] O. Babelon, C. M. Viallet ; Phys. Lett. B237 (1990), 411.

[9] A.M. Perelomov ; Sov. Journ. Nucl. Part. 10 (1979), 336.

[10] J. Avan, A. Jevicki; Comm. Math. Phys. (1992), to appear.

[11] M.A. Olshanetsky, A.M. Perelomov ; Inv. Math. 37 (1976), 93.

[12] V.I. Arnol'd ; "Mathematical Methods in Classical Mechanics" ; Graduate Texts in Mathematics 60, Springer Verlag.

[13] J. Moser ; Adv. Math. 16 (1975), 197.

[14] A.M. Perelomov ; Lett. Math. Phys. 2 (1977), 89.

[15] A.M.Perelomov ; Theor. Math. Phys. 6 (1971), 364 .

[16] L. Brink, T. Hanson, M.A. Vasiliev ; Phys. Lett. B286 (1992), 109.

[17] E.K. Sklyanin ; Preprint LOMI E-3-79 (1979).

[18] E.K. Sklyanin, Funct. Anal. Appl. 16 (1982), 27.

[19] I. Bakas; Comm. Math. Phys.134 (1990), 487.

[20] D. Olive, N. Turok; Nucl. Phys.B220 FS8 (1983), 491. 